\title{
Technology support for the inclusion of deaf students in mainstream schools: a summary of research from 2007 to 2017
}

\author{
Vaso Constantinou ${ }^{1}(1) \cdot$ Andri loannou $^{1} \cdot$ losif Klironomos $^{2} \cdot$ Margherita Antona $^{2} \cdot$ Constantine Stephanidis ${ }^{2,3}$
}

๑) Springer-Verlag GmbH Germany, part of Springer Nature 2018

\begin{abstract}
The paper examines the use of technology as a means for the inclusion of Deaf and Hard of Hearing (DHH) students in mainstream K-12 schools, covering the publications of the last decade. A systematic search of databases was carried out to locate theoretical and empirical studies investigating technologies as auxiliary or supporting tools for the inclusion of DHH students. Results are presented in two categories: (1) review/survey or theoretical papers and (2) studies of technology design and evaluation. Based on our findings, there is only preliminary evidence on how technology can support the inclusion of DHH students in mainstream schools. We argue that there is less need for research on technologies specialized on the disability, aiming to accommodate hearing loss, such as technologies to support the acquisition of sign language or speech-to-text translation of the teacher's talk. There is a major need for technology integration and research on its real-world use and utility, towards the inclusion of all students in the mainstream classroom, serving the needs of both DHH students and the general student population.
\end{abstract}

Keywords Deaf $\cdot$ Hard of hearing $\cdot$ Hearing impaired $\cdot$ Mainstream education $\cdot$ Inclusion $\cdot$ Technology-enhanced learning

\section{Introduction}

In recent years, inclusive education has developed into a global policy movement which advocates the rights of disabled students to receive access and quality participation in

Vaso Constantinou

va.constantinou@edu.cut.ac.cy

Andri Ioannou

andri.i.ioannou@cut.ac.cy

Iosif Klironomos

iosif@ics.forth.gr

Margherita Antona

antona@ics.forth.gr

Constantine Stephanidis

cs@ics.forth.gr

1 Cyprus Interaction Lab, Department of Multimedia and Graphic Arts, Cyprus University of Technology, 30 Archbishop Kyprianou Str., 3036 Lemesos, Cyprus

2 Foundation for Research and Technology-Hellas (FORTH), Institute of Computer Science, N. Plastira 100, Vassilika Vouton, 70013 Heraklion, Crete, Greece

3 Department of Computer Science, University of Crete, Heraklion, Crete, Greece mainstream classrooms [3]. UNESCO has determined that school education is for $a l l$; people with disabilities should not be marginalized from the formal education system due to their disability, but instead, should be offered equal opportunities for professional development and lifelong learning [31]. As a result of this movement, inclusive education (or inclusion) has become a global issue of considerable attention in the context of education policy, research and practice. A few works have been featuring this effort; from very specific approaches such as advocating the access and quality of participation of children with special educational needs in mainstream classrooms [21] to broader attempts aiming at educational inclusion as a means for creating an inclusive society [3]. The present review focuses on the former, with an emphasis on the inclusion of Deaf and Hard of Hearing (DHH) students in mainstream K-12 schools.

Mainstreaming DHH students affords challenges for all stakeholders, including teachers, DHH students, hearing peers, parents, and policy makers. In mainstream schools DHH students are often placed in oral programs that do not meet their needs, educationally, socially or emotionally, but rather exclude their right to supportive learning environments that enable, for instance, visual access to education [15]. On the one hand, in most mainstream classrooms sign 
language is not an option, even though lessons rely on verbal information and student-teacher communication. This means that the teachers and other student-peers not only have problems communicating with DHH students, but cannot understand them either $[9,10]$. On the other hand, when a sign language professional is available in the mainstream classroom, the interpreter is often not a teacher and, therefore, may not be able to interpret the teacher's lessons properly for the student, resulting again in poor communication $[16,30]$. Considering that verbal interaction amongst students and their teacher is the most important aspect of a classroom environment, it becomes apparent that DHH students miss out on this learning opportunity in the typical mainstream classroom. Along this line, a survey of the educational needs of DHH children in mainstream education in New Zealand, by Fitzgerald (2000), elaborated that mainstream services in schools do not work effectively for all DHH students [12]. They argued that there is a need for learning environments that make effective use of visual learning material, adaptive technology such as FM systems, and speech-to-text captioning, to accommodate hearing loss.

Last, but not least, with the progress in universal screening and technology of sensory aids (e.g., cochlear implants), a growing number of DHH students attend mainstream schools [18]. As a result, the educational system faces a very diverse population of DHH students with various degrees of abilities and who function neither as Deaf nor as hearing children [2]. These children use spoken language as the main way to communicate; yet, typically their reading level is lower than that of hearing students [32], whilst they exhibit weaknesses in the development of grammar [19]. There is compelling evidence that providing an appropriate educational environment for these students continues to be a challenge, especially in secondary and post-secondary settings, as the complexities of the learning domains and of the social environment increase. For example, De Raeve focused on education and rehabilitation for deaf children with cochlear implants and discussed the changes needed in the educational services. The author focused on the skills required by the teachers and staff, including flexibility, technology orientation and updates, and ongoing professional development to cover the learning and emotional needs of the children, providing an appropriate environment for their psycho-social needs. As the author explained, the challenge for the near future is to be able to address the needs of each child, and deliver services depending on their abilities, expectations, and attitudes [8]. Although the advantages of technological sensory aids are definitively clear, it is still necessary to consider the special needs of the population of DHH students and provide flexibility of choice in meeting those needs [2].

Overall, DHH students in the educational system have served as both the objective and the inspiration for many innovation studies through the years, which have increased the quality of life and professional opportunities for deaf people of all ages [28]. In today's mainstream classrooms, however, there seems to be underutilization of technology, whilst its potential to support the inclusion of DHH students remains relatively unexplored. To inspire new research in this area, the present review of the literature examines the use of technology, in the last decade, as a means for the inclusion of DHH students in mainstream K-12 schools. The study reports on theoretical and empirical studies which consider technologies as auxiliary or supporting tools for the education of DHH children, whilst identifying gaps and directions for future research. This work is situated in an 'open' space for academic inquiry within the broader area of $\mathrm{HCI}$, human factors, and technology advancements in special education; the methodology and results are discussed next, concluding with a call for more empirical research in specific directions.

\section{Methodology}

The review of the literature covers studies published in English, between 2007 and 2017. The process involved three steps, as follows.

Step 1: Database search A search was conducted in the following electronic databases, which were regarded as possible venues for educational research: EdITLib, ACM Digital Library, Emerald, ERIC, JSTOR, Proquest, Sage, Science Direct, Scopus, Springer Link, Taylor and Francis Online. Also, we used the Google Scholar search engine. The search keywords, by looking into authors' keywords and abstract, were (technolog*) AND ((deaf OR "hard of hearing")) AND (((mainstream* OR (special AND ed*) OR Inclus*))). The database search yielded 176 manuscripts which were published from 2007 to 2017.

Step 2: Study selection The selection of studies was based on specific criteria: (1) addressing some form of technology use, (2) clear link to DHH education/learning, (3) clear concern with mainstreaming deaf students. One researcher carefully inspected titles and abstracts of all manuscripts in the pool and excluded all papers which did not match the criteria. The excluded papers were reviewed independently by a second researcher who confirmed (with $100 \%$ agreement) that the criteria were correctly applied. In the end, 159 documents were excluded and 17 remained in the pool.

Step 3: Synthesis Both authors inspected the content of the resulting 17 manuscripts and synthesized the findings, grouped into two categories: (1) reviews/ surveys or theoretical works (e.g., discussion of models), (2) studies of technology design and evaluation. In the following section, an analysis of these manuscripts is presented. 


\section{Results}

\subsection{Theoretical or review/survey studies}

A few theoretical and review studies published on the issue of technology support of deaf education in mainstream classrooms, in the last decade (see Table 1 for an overview) were identified. For example, a previous review by Lidströmn and Hemmingsson was based on 32 review articles, 16 of which were intervention studies. The authors found that, regardless of the type of technology, the use of ICTs supported students with special needs, including DHH students, particularly in the areas of writing, spelling and communication. The authors discussed the need for more research and intervention studies concerning students with visual, hearing, and communication impairments [20]. In another review paper, speech-to-text technology was found to be a particularly promising tool for the inclusion of DHH students [26]. These authors found that using speech-to-text in traditional learning environments helped DHH students understand the learning content and enabled them to take notes, complete homework, and prepare for exams [26]. Some major concerns were raised by Beal-Alvarez and Cannon arguing that the quality indicators need more attention in studies of DHH education. In their work, 29 peer-reviewed studies (published in 2000-2013) were examined, addressing technologybased interventions for DHH students aimed at academic gains. The authors found that only a few studies satisfied a list of predefined quality indicators for individual intervention studies, concluding that replication of intervention studies is needed [5].

In the context of mobile learning, Nathan et al. elaborated on a usability evaluation model applied to the design of mobile applications for DHH people. They suggested that future work should focus on establishing guidelines for the design of mobile applications for DHH education [23]. Furthermore, other researchers have argued that more research is needed in the area of online learning and the types of practices that make inclusion possible. For instance, Burgstahler's review documented the various characteristics of distance learning programs that might improve the inclusion of students with disabilities [6]. Moreover, comparing with traditional ways of teaching, Shepherd and Alpert's review discussed how online learning can support DHH students better than traditional learning environments; the authors discussed how technological modalities in online learning were more attractive, motivated DHH learner's activity, and expanded their visual creativity and intelligence [27].

\subsection{Studies on technology design and evaluation}

Mobile applications appear to be widely used with mainstreamed DHH students in the last decade, as illustrated in Table 2. Most of these studies sought to support DHH students in learning the sign language, targeting the improvement of communication between DHH students and their parents and teachers $[1,7,13,17,22,24,29]$. A number of applications were designed to present vocabulary or phrases in sign language, helping students to develop spelling and literacy skills. For example, Chuan and Guardino discussed a design called "SmartSignPlay", which is an interactive mobile application. The application supports learning and practice of the American Sign Language (ASL) using signs through an animated avatar. Its usability was evaluated by five hearing children under the age of 10 and two hearing adults, with inconclusive results in terms of effectiveness in learning ASL [7]. Similarly,

Table 1 Review/survey or theoretical works

\begin{tabular}{|c|c|c|c|}
\hline & Technology & Manuscript details & Author \\
\hline 1 & Mobile application & Discussion of evaluation models for mobile applications for the DHH & Nathan et al. [23] \\
\hline 2 & Online learning & Review (2005-2012) of e-learning environments for DHH students & Hashim et al. [14] \\
\hline 3 & Online learning & $\begin{array}{l}\text { Documentation of the characteristics of distance learning programs } \\
\text { addressing social inclusion of DHH students }\end{array}$ & Burgstahler [6] \\
\hline 4 & Online learning & $\begin{array}{l}\text { Presentation of how technological modalities in online learning can } \\
\text { differentiate instruction for DHH students }\end{array}$ & Shepherd and Alpert [27] \\
\hline 5 & Speech-to-text recognition & $\begin{array}{l}\text { Review (1999-2014) of speech-to-text recognition technology applied } \\
\text { to the education of the DHH }\end{array}$ & Shadiev et al. [26] \\
\hline 6 & Interactive whiteboards & $\begin{array}{l}\text { Review (2000-2013) of results from the integration of interactive } \\
\text { whiteboards in special (including DHH) education }\end{array}$ & Drigas and Papanastasiou [11] \\
\hline 7 & Technology-based interventions & $\begin{array}{l}\text { Review of peer-reviewed studies (2000-2013) on technology-based } \\
\text { interventions with DHH children }\end{array}$ & Beal-Alvarez and Cannon [5] \\
\hline 8 & ICT school activities & $\begin{array}{l}\text { Review (2000-2012) of the types of ICT tools being used by DHH } \\
\text { students }\end{array}$ & Lidström and Hemmingsson [20] \\
\hline
\end{tabular}


Table 2 Studies of technology design and evaluations

\begin{tabular}{|c|c|c|c|}
\hline & Technology & Manuscript details & Author \\
\hline 1 & Mobile app & $\begin{array}{l}\text { Learn and practice ASL: "SmartSignPlay" mobile app teaches vocabulary and phrases in } \\
\text { ASL through an animated avatar }\end{array}$ & Chuan and Guardino [7] \\
\hline 2 & Mobile app & $\begin{array}{l}\text { Learn foreign sign languages: prototype mobile application to support four foreign sign } \\
\text { languages through Video clips from native signers }\end{array}$ & Parton [24] \\
\hline 3 & Mobile app & $\begin{array}{l}\text { Allow students' active participation and teacher feedback and assessment } \\
\text { A mobile application to teach language, a 2D representation of WhatsApp messaging }\end{array}$ & Magon et al. [22] \\
\hline 4 & Mobile app & $\begin{array}{l}\text { Facilitate knowledge exchange and improve shared understanding among hearing and } \\
\text { non-hearing people: Arabic sign language videos (contain a dictionary, tutorial, and } \\
\text { fingerspelling editor) }\end{array}$ & Al-Nafjan et al. [1] \\
\hline 5 & Mobile app & $\begin{array}{l}\text { Develop fingerspelling and literacy skills: 3D fingerspelling mobile app, called "Finger- } \\
\text { spelling Tutor" }\end{array}$ & Toro et al. [29] \\
\hline 6 & Mobile app & $\begin{array}{l}\text { Improve communication and access to information: mobile app using Text-to-Arabic sign } \\
\text { language via 3D animations and feedback about the meaning of the Arabic text }\end{array}$ & Halawani [13] \\
\hline 7 & Mobile app & Enable communication: prototype app of real-time captioning on tablet & Iglesias et al. [17] \\
\hline 8 & Game & Learn and practice ASL: game "CopyCat" with gloves to help teach ASL & Zafrulla et al. [33] \\
\hline 9 & Game & $\begin{array}{l}\text { Enhance the strategies of acquiring knowledge: game for language learning (connect the } \\
\text { dots, jigsaw puzzle, word/image association, drawing, painting, etc.) }\end{array}$ & Portugal and de Souza Couto [25] \\
\hline
\end{tabular}

Toro et al. presented an application called "Fingerspelling Tutor" which uses a 3D animated character to demonstrate fingerspelling. The application includes quizzes and practice lessons allows the user to type in words that a $3 \mathrm{D}$ character can fingerspell, and connects to social media to help create a virtual community among DHH users. The app was found to support the development of literacy skills [29].

Other efforts focused on facilitating communication between DHH students and their teachers in the mainstream classroom. First, Magon et al. aimed at the active participation of DHH students by activating feedback and evaluation of teachers via a mobile application. The so-called, "WhatSurdo", was a messaging application similar to WhatsApp for real-time communication. The application was tested with four DHH students. The authors argued that Whatsurdo supported teachers who taught language to DHH students of different ages [22]. Second, Iglesias et al. demonstrated a method of inclusive education using a speech-to-text captioning technology. APEINTA, transcribed a classroom teacher's spoken words into captions, enabling communication not only for both DHH students but also foreign students who did not speak the language [17].

Furthermore, there is some work on computer games (see Table 2), aimed at language learning for DHH students [25, 33]. For example, Portugal and de Souza Couto described the design of a multi-track game to support the development of a language for DHH children [25]. Also, a study by Zafrulla et al. employed the use of special coloured gloves in the so-called CopyCat game to help teach ASL. The study tested the recognition of phrases by the children and demonstrated benefits in terms of real-time ASL phrase recognition [33].

\section{Discussion}

This summary of research examined the use of technology as a means for the inclusion of DHH students in mainstream K-12 schools, covering publications of the last decade. After careful examination in a number of online bibliographic databases, only few empirical studies appear to address the use of technology to support the inclusion of DHH students in mainstream schools. Research in this area appears to be limited, with only 17 publications meeting our criteria (clear link to DHH students and their mainstreaming) across a wide range of databases. Despite the overall effort, there are some major issues regarding research in this context, which are discussed next.

Although there are a few efforts addressing the design of applications for the support of DHH students-mainly mobile apps and games-more than half of these studies do not provide data from research evaluations [1, 13, 17, $25,29]$. Other studies provide very limited data on prototypes of applications (see studies reported earlier with 2-4 participants) leading to only anecdotal evidence of the effectiveness of the technology. It is also very puzzling that, when some form of evaluation does exist, the authors do not report the context of the evaluation (i.e., details of the mainstream classroom, role of teacher and peers). Last but not least, none of the empirical studies of this review reports on the level of hearing loss of the participants, making it difficult to assess the needs of the participants under study, with respect to the technology used. In particular, the studies reported here made no reference to the diverse abilities and needs of the participating DHH students [2], while the ways in which technology targeted their inclusion were not adequately addressed. 
Overall, our review provides only initial evidence on how the technology can support the inclusion of DHH students. Further research is needed in the area to address some of the problems that mainstreamed DHH students' face. Future research should focus on individual intervention studies with high-quality indicators [5] and should always report on the level of hearing loss of the participants, the context of the study (e.g., details of the mainstream classroom), and any other information that makes apparent the added value of the technology.

Given the current technological advancements, there is great potential to conduct technology research, targeting inclusion and proper education for DHH students by enabling positive social interactions and collaboration with their hearing peers [4]. What these technologies might look like and how to best design and utilize them remain 'open' questions for academic inquiry within the broader area of HCI, human factors, and technology advancements for special education. We would argue that less research is needed on the aspects of technological development and laboratory evaluations; instead, more research on technology integration and its real-world use and utility in the mainstream classroom is necessary. In other words, rather than prototype applications and isolated evaluations, future research might focus on providing platforms and tools that teachers can adapt to the needs of their students and of the setting. Technology research should focus on supporting the educators in addition to the DHH student.

Closing, the current literature provides very limited knowledge and information for researchers and practitioners who aim to integrate technology in the mainstream classroom to improve learning for DHH students and for all. The results of this review suggest that we are still in very early stages of technology research aimed at the integration of DHH students (and potentially other students with special educational needs) in the mainstream schools. We suggest that there is immediate need for research in this area. Such research is of paramount importance when one considers the increase in the number of students with special needs in mainstream schools worldwide vis-à-vis the underutilization of technology in the classroom.

Acknowledgements Authors acknowledge funding from the European Union's Horizon 2020 Framework Programme through NOTRE project. This project has received funding from the European Union's Horizon 2020 research and innovation programme under Grant Agreement no. 692058 .

\section{References}

1. Al-Nafjan, A., Al-Arifi, B., Al-Wabil, A.: Design and development of an educational Arabic sign language mobile application: collective impact with Tawasol. In: Antona, M., Stephanidis,
C. (eds.) Universal access in human-computer interaction, vol. 9176, pp. 319-326. Springer, Cham (2015). https://doi. org/10.1007/978-3-319-20681-3_30

2. Archbold, S., Mayer, C.: Deaf education: the impact of cochlear implantation? Deaf. Educ. Int. 14(1), 2-15 (2012)

3. Barton, L., Armstrong, F. (eds.): Policy, Experience and Change: Cross-cultural Reflections on Inclusive Education, vol. 4. Springer, Dordrecht (2007)

4. Batten, G., Oakes, P.M., Alexander, T.: Factors associated with social interactions between deaf children and their hearing peers: a systematic literature review. J. Deaf Stud. Deaf Educ. 19(3), 285-302 (2014)

5. Beal-Alvarez, J., Cannon, J.E.: Technology intervention research with deaf and hard of hearing learners: levels of evidence. Am. Ann. Deaf. 158(5), 486-505 (2014). https://doi. org/10.1353/aad.2014.0002

6. Burgstahler, S.: (2015). Opening doors or slamming them shut? Online learning practices and students with disabilities. Soc. Incl. 3(6). https://doi.org/10.17645/si.v3i6.420

7. Chuan, C.H., Guardino, C.A.: Designing SMARTSIGNPLAY: an interactive and intelligent american sign language app for children who are deaf or hard of hearing and their families. In: Companion publication of the 21 st international conference on intelligent user interfaces, pp. 45-48. ACM, New York (2016). https://doi.org/10.1145/2876456.2879483

8. De Raeve, L.: Education and rehabilitation of deaf children with cochlear implants: a multidisciplinary task. Cochlear Implants Int. 11(suppl 1), 7-14 (2010)

9. DeWitt, D., Alias, N., Ibrahim, Z., Shing, N.K., Rashid, S.M.M.: Design of a learning module for the deaf in a higher education institution using padlet. Procedia Soc. Behav. Sci. 176, 220-226 (2015). https://doi.org/10.1016/j.sbspro.2015.01.464

10. Ditcharoen, N., Naruedomkul, K., Cercone, N.: SignMT: an alternative language learning tool. Comput. Educ. 55(1), 118 130 (2010). https://doi.org/10.1016/j.compedu.2009.12.009

11. Drigas, A.S., Papanastasiou, G.: (2014). Interactive white boards' added value in special education. Int. J. Online Eng. 10(6). https://doi.org/10.3991/ijoe.v10i6.4004

12. Fitzgerald, T.: The educational needs of deaf and hearing impaired children. Specialist Education Services, Wellington (2000)

13. Halawani, S.M.: Arabic sign language translation system on mobile devices. IJCSNS Int. J. Comput. Sci. Netw. Secur. 8(1), 251-256 (2008)

14. Hashim, H., Tasir, Z., Mohamad, S.K.: (2013). E-learning environment for hearing impaired students. TOJET: Turk. Online J. Educ. Technol. 12(4):67-70

15. Human Rights-WFD. WFD. (2016) https://wfdeaf.org/human -rights/. Retrieved 22 April 2017

16. Hyde, M., Punch, R., Power, D., Hartley, J., Neale, J., Brennan, L.: The experiences of deaf and hard of hearing students at a Queensland University: 1985-2005. Higher Educ. Res. Dev. 28(1), 85-98 (2009). https://doi.org/10.1080/07294360802444388

17. Iglesias, A., Ruiz-Mezcua, B., López, J.F., Figueroa, D.C.: New communication technologies for inclusive education in and outside the classroom. In: Assistive technologies: concepts, methodologies, tools, and applications. IGI Global (2013). https://doi. org/10.4018/978-1-4666-2530-3.ch013

18. Kelman, C.A., Branco, A.U.: (Meta) Communication strategies in inclusive classes for deaf students. Am. Ann. Deaf 154(4), 371-381 (2009)

19. Lederberg, A.R., Schick, B., Spencer, P.E.: Language and literacy development of deaf and hard-of-hearing children: successes and challenges. Dev. Psychol. 49(1), 15 (2013)

20. Lidström, H., Hemmingsson, H.: Benefits of the use of ICT in school activities by students with motor, speech, visual, 
and hearing impairment: a literature review. Scand. J. Occup. Ther. 21(4), 251-266 (2014). https://doi.org/10.3109/11038 128.2014.880940

21. Lipsky, D.K., Gartner, A.: Inclusion and school reform: transforming America's classrooms. Paul H. Brookes Publishing, Baltimore (1997)

22. Magon, D.P.D.S., Campello, A.R.E.S., Castro, H.C.: WhatSurdo: a strategy to simulate the real communicational world in low income schools. Sch. Int. J. Multidiscip. Allied Stud. 3(4), 76-82 (2016). https://doi.org/10.19085/journal.sijmas030401

23. Nathan, S.S., Hashim, N.L., Hussain, A.: Usability evaluation of mobile application: a review on usability model for the deaf. J. Teknol. 78(4-3), 127-131 (2016)

24. Parton, B.: Facilitating exposure to sign languages of the world: the case for mobile assisted language learning. J. Inf. Technol. Educ. Innov. Pract. 13:13-24 (2014)

25. Portugal, C., de Souza Couto, R.M.: Designing a learning game for the deaf children as an educational technology. Contemp. Educ. Technol. 3(1):60-75 (2012)

26. Shadiev, R., Hwang, W.Y., Chen, N.S., Yueh-Min, H.: Review of speech-to-text recognition technology for enhancing learning. J. Educ. Technol. Soc. 17(4), 65 (2014)

27. Shepherd, C.M., Alpert, M.: Using technology to provide differentiated instruction for deaf learners. J.Instr. Pedag. 16, 1 (2015)
28. Spaulding, L.S., Pratt, S.M.: A review and analysis of the history of special education and disability advocacy in the United States. Am. Educ. Hist. J. 42(1/2), 91 (2015)

29. Toro, J.A., McDonald, J.C., Wolfe, R.: Fostering better deaf/hearing communication through a novel mobile app for fingerspelling. In: Miesenberger, K., Fels, D., Archambault, D., Peňáz, P., Zagler, W. (eds.) Computers helping people with special needs. ICCHP 2014, vol. 8548, pp. 559-564. Springer, Cham (2014). https://doi. org/10.1007/978-3-319-08599-9_82

30. Traynor, N., Harrington, F.: BSL/English interpreting in higher education: is access to the curriculum a reality for deaf students? In: Galloway, C., Young, A. (eds.) Deafness and education in the UK: Research perspectives, pp. 205-239. Whurr, Chichester, England (2003)

31. UNESCO: Guidelines for Inclusion: Ensuring Access to Education for All. UNESCO, Paris (2005)

32. Vermeulen, A.M., Van Bon, W., Schreuder, R., Knoors, H., Snik, A.: Reading comprehension of deaf children with cochlear implants. J. Deaf Stud. Deaf Educ. 12(3), 283-302 (2007)

33. Zafrulla, Z., Brashear, H., Yin, P., Presti, P., Starner, T., Hamilton, H.: American sign language phrase verification in an educational game for deaf children. In: 2010 20th international conference on pattern recognition (ICPR), pp. 3846-3849. IEEE (2010). https ://doi.org/10.1109/ICPR.2010.937 